\title{
Some Considerations Regarding Mathematical Semiosis
}

\author{
Ricardo Gudwin and João Queiroz
}

possible solution to this problem would be to use the Peircean model of meaning, given by the theory of semiotics from the philosopher Charles Sanders Peirce. We have been working with Peirce's theory of sign, trying to bring his model into the theory of intelligent systems [2-8].

The main motivation for this work is to model the Peircean concept of "meaning process" in an entirely mathematical framework, creating what we call "Mathematical Semiosis". We may understand this strategy as a kind of "transposition" of the Peircean model of semiosis to a mathematical model, which we consider to be more interesting for the engineering point of view. Our goal is to bring a more understandable theory for explaining "meaning" in a strictly mathematical sense. It is mainly a theoretical work, but with a possible great impact in the practical construction of artificial systems able to find meaning in its surrounding environment.

\section{II.MEANing ACCORDING TO PEIRCE}

Peirce identify meaning as a special kind of process which The creation of (multi) agent systems able to fully interpret its environment is one of the big dreams of artificial intelligence. This problem proved to be much more complicated than could be imagined at once, and to the extension this challenge became more acknowledgeable, gradually this dream gave rise to more modest claims, paving the road for what is currently the technology of Intelligent Systems. One of the problems related to achieving the aforementioned dream is the lack of good framework (or too many inadequate models) for modeling "meaning" and “interpretation”. According to Deb Roy, 'A grand challenge for the cognitive sciences is to develop a computational framework that simultaneously models referential and functional meaning' ('Meaning Machines'; available at: http://web.media.mit.edu/ dkroy). Some recent efforts, based on semiotic considerations, are trying to address this issue in a more proper manner [1]. The requirement to involve "reality" in this discussion, brings forth a lot of unsolved questions regarding the nature of "reality". We understand that a

Manuscript received March 30, 2007. This work was supported in part by grants from CNPq and FAPESP, the Brazilian National Council for Research, and the Foundation for Supporting Research of the State of São Paulo.

R. Gudwin is with the Department of Computer Engineering and Industrial Automation, Faculty of Electrical and Computer Engineering, State Univesity of Campinas, DCA-FEEC-UNICAMP, Av. Albert Einstein 400, 13083-852, Campinas - SP, Brazil (phone +55-19-3521-3819, fax: +55-193521-3845; e-mail: gudwin@dca.fee.unicamp.br).

J. Queiroz is with the Department of Computer Engineering and Industrial Automation, Faculty of Electrical and Computer Engineering, State University of Campinas, DCA-FEEC-UNICAMP, Av. Albert Einstein 400, 13083-852, Campinas - SP, Brazil (e-mail: queirozj@dca.fee.unicamp.br)

\begin{abstract}
The discussion on the possibility of machines to
achieve semiosis (meaning process) is a very controversial debate within Artificial Intelligence and Cognitive Science. In this work, framework. We call the "transposition" of the Peircean theory to an abstract mathematical model as "Mathematical Semiosis". By explaining what is to mean, in a strictly mathematical sense, avoiding complications related to the connection of signs to a real to develop machines with these capabilities. In such a regard,
\end{abstract}

Index Terms — Semiosis, Meaning, Mathematical Semiosis.
“... by 'semiosis' I mean [...] an action, or influence, which is, or involves, a cooperation of three subjects, such as a sign, its object, and its interpretant, this tri-relative influence not being in any way resolvable into actions between pairs". (CP 5.484)

Peirce conceives a 'Sign' or 'Representamen' as a 'First' which stands in such a genuine triadic relation to a 'Second', called its 'Object', so as to be capable of 'determining a Third', called its 'Interpretant', to assume the same triadic relation to its Object in which it stands itself to the same Object. To cite him:

"My definition of a sign is: A Sign is a Cognizable that, on the one hand, is so determined (i.e., specialized, bestimmt) by something other than itself, called its Object, while, on the other hand, it so determines some actual or potential Mind, the determination whereof I term the Interpretant created by the Sign, that that Interpreting Mind is therein determined mediately by the Object" (CP 8.177).

Another important concept is the notion of Peirce's logicalphenomenological categories. For Peirce, reality can be decomposed into processes that should fit into just three different categories, which he calls firstness, secondness and thirdness. In brief, the categories can be defined as: (1) Firstness: what is such as it is, without reference to anything else; (2) Secondness: what is such as it is, in relation with 
something else, but without relation with any third entity; (3) Thirdness: what is such as it is, insofar as it is capable of bringing a second entity into relation with a first one in the same way that it brings itself into relation with the first and the second entities. Firstness is the category of vagueness, freedom, novelty and originality - 'firstness is the mode of being which consists in its subject's being positively such as it is regardless of anything else. That can only be a possibility' (CP 1.25). Secondness is the category of reaction, opposition, differentiation, existence - 'generally speaking genuine secondness consists in one thing acting upon another, - brute action' [...] 'I consider the idea of any dyadic relation not involving any third as an idea of secondness' (CP 8.330). Thirdness is the category of mediation, habit, generality, growth, and conceptualization or cognition (CP 1.340). In another way of putting the categories: Firstness is possibility, what might become, Secondness is what is taken to be what is within some particular context, and Thirdness is what in all probability would be, given a certain set of conditions.

\section{RELATED APPROACHES AND BACKGROUND}

Other mathematical tentative approaches were conducted e.g. by Marty [9-12], by Goguen [13-15], by Burch [16,17] and by ourselves [2,3], but with different purposes.

Burch $[16,17]$ is concerned mainly on what he calls PAS Peircean Algebraic Logic, which is a relational algebraic system of logic formalizing and modifying some of the logical ideas of Peirce. His main result is the proof of Peirce's Reduction Thesis. This thesis states that relations of adicity 2 may not in general be constructed from relations exclusively of adicity 1 , that relations of adicity 3 or greater may not in general be constructed from relations exclusively of adicities 1 and/or 2, and that all relations, regardless of the domain of arbitrary (non-negative integers) adicities may be constructed from relations exclusively of adicities 1, 2 and 3 . The proof of this thesis is fundamental in order to sustain Peirce's category theory and Burch results are very important ones.

Goguen [13-15], on the contrary, explicitly claims to be developing a mathematically precise theory of semiotics, which he calls Algebraic Semiotics. His approach, though, is not purelly Peircean, and at the same time he uses some of Peirce's notions, he also includes Saussure's notions in a mixed framework. His strategy is to build what he calls sign systems, which are algebraic theories with extra structure, and also semiotic morphisms, which are mappings of algebraic theories that preserve the extra structure. Goguen claims to be developing his own general theory of meaning. He applies his theory mainly in the field of user interface design. Despite the complexity of his mathematical definitions, it seems that Goguen's theory is not as general and inclusive as Peirce's model for semiosis.

Marty [9-12], on the other side, clearly claims to be giving a formal treatment of the semiotic phenomena, extending Peirce's works in phenomenology and semiotics. He proposes a general methodology for formalizing the functioning of every system of signs, using essentially category theory and functors. Marty's approach follows the same insight we are using here, trying to evolve a formal perception theory, which generalizes to a full mathematical description of semiosis. His approach is based on typed relational structures, which gives rise to be classified under different formal categories. Under certain conditions these relational structures are defined as phenomenological structures, and based on possible morphisms on these, he defines phenomenological morphisms. Based on the set of possible phenomenological morphisms, he defines a possible set of six fundamental modes of being, which he correlates to the Peircean cenopythagorean categories and their degenerated forms. He then defines formally a phaneron as a diagram in a phenomenological category. Finally, he uses these phanerons in order to model the substitution of a phenomenon by another, giving rise to a representation, or a semiotic process. Marty's mathematical model is sophisticated, and he tries to follow Peirce's theory with precision. But his model is very complicated in mathematical terms to facilitate the connection between semiotics and Artificial Intelligence.

Our mathematical model of semiosis is actually based on some key notions:

- Peircean semiosis

- Cellular Automata

- Rosen's Anticipatory Systems Theory

\section{A. Cellular Automata}

Cellular Automata [18] comprises a discrete model studied in computability theory, mathematics, and theoretical biology, consisting of a (potentially) infinite, regular grid of cells, each one in a finite number of states. The grid may have any finite number of dimensions. Time is also discrete, and the state of a cell at time $t$ is a function of the states of a finite number of cells (called its neighborhood) at time $t$ - 1 . These neighbors are a selection of cells relative to the specified cell (which may include the own cell), and usually do not change. Every cell has the same rule for updating, based on the values of states in this neighborhood. Each time the rules are applied to the whole grid a new generation is created. An example of a three-dimensional cellular automaton is given in figure 1. In figure 1 , we have a three-dimensional grid of states, and the

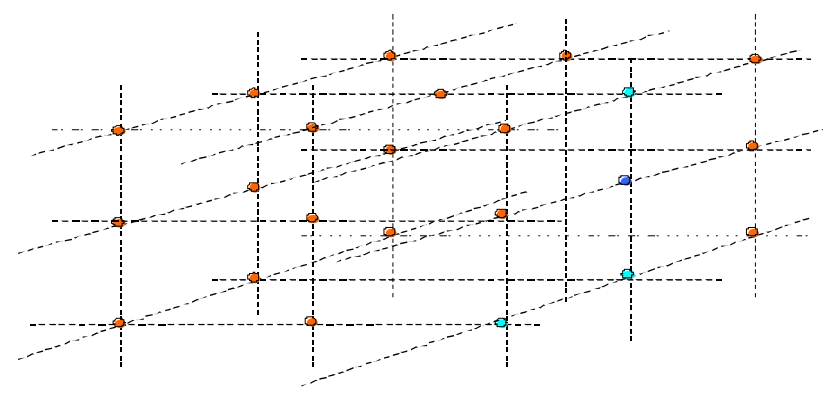

Figure 1 - A 3D Cellular Automaton 
values of these states are correlated in time. In the figure, the value of state $\mathrm{S}$ in instant $t+1$ is a function $f$ of the values of states in its neighborhood neigh $(S)$ in an instant $t$.

This concept is useful here because we will use it in order to define our mathematical reality as a kind of cellular automata.

\section{B. Anticipatory Systems}

An anticipatory system is a system whose current state is determined by a future state, or, according to Robert Rosen [19]:

"A system containing a predictive model of itself and/or its environment, which allows it to change state at an instant in accordance with the model's predictions pertaining to a latter instant."

These predictions can be goals, plans or simply estimations of future states.

Acording to Mihai Nadin [20,21]:

"Anticipation is a recursive process described through the functioning of a mechanism whose past, present, and future states allow it to evolve from an initial to a final state that is implicitly embedded in the mechanism".

Anticipatory systems are very different from the standard kind of systems we are used to find in engineering and systems sciences, and have many interesting properties that make them more than pure mechanical deterministic systems. Rosen argues that their behavior is what make living systems different from non-living systems. Living systems would be anticipatory systems. Anticipatory systems may provide also the kind of teleological behavior that is particularly related to the property we use to call "intelligence" in human beings.

This teleological behavior situate anticipatory systems as a natural candidate to instantiate the Peircean notion of "thirdness". Peirce argues that all that can be known must fit into three different categories: firstnesses, secondnesses and thirdnesses. This is in the kernel of Peircean philosophy. But how to understand Peirce's claims if we assume very simple systems as our reality?

A simple system like

$$
S(t+1)=\operatorname{Random}()
$$

will be a system of pure firstness. Supposing that $t+1$ is equivalent to the present time, we have a system where the present is completely random.

A system like

$$
S(t+1)=f(S(t))
$$

will be a purely deterministic, or mechanical system. This is a system where there is only secondness. It is a system where the present is completely determined by the past.

A system like

$$
S(t+1)=f(S(t))+\operatorname{Random}()
$$

will be a system where there is firstness and secondness.

A candidate for a system with thirdness will be something like:

$$
S(t+1)=f(S(t+\tau))
$$

or, in other words, a system where the present depends on the future. But, if time evolves from the past to present, this seems to be impossible. How can this be possible ? To understand that, we need to make a change in the equation:

$$
S(t+1)=f(E(S(t+\tau))(t))
$$

In this case, $E($.$) is the estimation in time t$ of a future state $S(t+\tau)$. This system is perfectly feasible. But, $f(E(S(t+\tau))(t))$ can be rewritten to $f_{2}(S(t))$, and then it simply reduces again to a deterministic system. So, this is clearly not the answer. How to still have a true anticipatory system ? The solution is to provide an open system instead of a closed one:

$$
S(t+1)=f(S(t))+g(U(t))
$$

where $U(t)=S(t+\tau)$ is an external input.

But, if $S(t)$ is our reality, requiring an external input out of this reality to explain its states, someone would argue that to assume the existence of $U(t)$ is equivalent to accept a dualist position (in cognitive science). But, if we accept that the $S(t)$ is just our sensorial reality (and not the whole physical reality), there may be something else in physical reality that is not in sensorial reality, and we may argue that this something else is responsible for the anticipatory component of the system, so both a dualist and a non-dualist explanation are possible. Our only claim is that the anticipatory behavior is due to something out of sensorial reality, just this. At this point, though, we are not making further assumptions on how this anticipatory behavior is possible.

With this brief introductory background, we are now prepared to our definitions.

\section{A Mathematical Model of Semiosis}

We define in this session a general mathematical framework for Peircean semiosis. Nevertheless, our intention with this is to allow a better appreciation by the engineering community of the potentialities of the Peircean model of representation in order to build agents able to perform semiosis.

In our general framework, we define a Mathematical Universe $U$ which is composed of a Mathematical Reality $R$ and a set of agents $A=\left\{A_{i}\right\}$.

$$
U=(R, A)
$$

The Mathematical Reality $\mathrm{R}$ is defined as

$$
R: P \times T \rightarrow V
$$

where $R$ is a function which returns a value $v \in V$ to each 
place $p \in P$ ( $p$ is a coordinate in an n-dimensional grid) and time $t \in T$. At this point of the formalization, we will not impose any restriction on set $V . V$ can be regarded as a set of possible states which each part of reality $\mathrm{R}$ can assume. For the sake of an example, we may assume (e.g.) that $V=[0,1]$. Another example may be $V=[0,1]^{n}$ where $\mathrm{n}$ is the number of different measurings that someone is able to perform at a particular place and time. To avoid any limitation on the formalism, we will leave $V$ generic by this time.

The definition of $R$ is quite open, and serves our purpose of generality for the framework. To allow us a better understanding of a potential use of $R$, let us compare it to a cellular automaton. In order for the function $R$ to be a cellular automaton, this function should be written as:

$$
R(p, t+1)=f\left(R\left(q_{1}, t\right), \ldots, R\left(q_{m}, t\right)\right)
$$

where

$q_{i} \in \operatorname{Neigh}(p)$ and $\operatorname{Neigh}(p)$ is a set of places which comprises the neighborhood of place $p$. But this will make $R$ a purely deterministic system. In our case, we want our mathematical reality to be composed of firstness, secondness and thirdness processes, and so our definition for $R$ will be a little bit different. In our case our mathematical reality will be defined as:

$$
R(p, t+1)=R_{1}+R_{2}+R_{3}
$$

where

$$
\begin{gathered}
R_{1}=\operatorname{Random}() \\
R_{2}=f\left(R\left(q_{1}, t\right), \ldots, R\left(q_{m}, t\right)\right), q_{i} \in \operatorname{Neigh}(p) \\
R_{3}=\sum_{i} \operatorname{Act}\left(A_{i}, p, t\right)
\end{gathered}
$$

$\operatorname{Act}\left(A_{i}, p, t\right)$ is the contribution of Agent $A_{i}$ to the state on place $p$ in time $t$. This component is equivalent to $U(t)$ in equation (6). Component $R_{1}$ is a component of firstness. Component $R_{2}$ is a component of secondness and component $R_{3}$ is a component of thirdness. With this, our mathematical reality fully instantiates the Peircean notion of reality. The defined mathematical reality is illustrated in figure 2 .

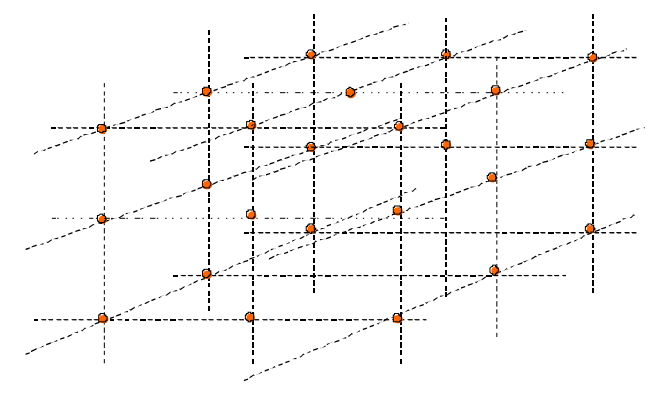

Figure 2 - Mathematical Reality Actuated by Agents
The other component of our mathematical universe $U$ is a set of Agents $A$. Each agent $A_{i}$ in $A$ is able to sense and actuate on mathematical reality $R$ (see figure 2). This means that it will be able to contribute to the new values of states within reality $R$, according to equation (10). This contribution may be a function of some states in reality $R$. But each agent is limited to just a limited subset of places in $P$, for sensing, and another subset of $P$ for actuation. These subsets may change in time. Let us define then the function $\Pi$ :

$$
\Pi: A \times T \rightarrow 2^{P}
$$

which we call the Perceptive Scope function, which defines for each $A_{i}$ and time $t$ a set of places which can be measured by the agent. As a complement, let us define the function $\Gamma$ :

$$
\Gamma: A \times T \rightarrow 2^{P}
$$

which we call the Actuative Scope function, which defines for each $A_{i}$ and time $t$ a set of places which can be actuated over by the agent.

Let us now make some simplifications in order to give some ground for the next development. Let us imagine that $\mathrm{P}$ is given by:

$$
P=\stackrel{n}{\times} Z
$$

where $Z$ is the set of integer numbers and $\mathrm{P}$ is the cartesian product of it $\mathrm{n}$ times. In this case, each $p=\left(p_{1}, \ldots, p_{n}\right)$ will be a place in an n-dimensional grid.

In this case, we will define a region $\mathrm{G}$ to be any subset of $\mathrm{P}$ :

$$
G \subset P
$$

We will then define an Attention Window $G^{S}$, to be a region generated by a given place and a set of rules for including other places into the region:

$$
G^{S}=S(p)
$$

where $S: P \rightarrow 2^{P}$ and $\mathrm{S}$ is a function which for a given place $\mathrm{p}$, determines a set of other places relative to $\mathrm{p}$, which may be a part of the region which is said to be located in place p. Usually $\mathrm{S}$ can be given by a script which given $\mathrm{p}$, generates the other places member of the region $G^{S}$.

We may now restrict our framework, such that every Perceptive Scope and Actuative Scope of every agent in our mathematical universe are Attention Windows. This means that the sources and sinks of information processed by agents will always have the same structure all around the mathematical reality.

Now, let us make some generalizations. Even though $\mathrm{R}$ is a function of single places $p$ in time t, we will use the same notation to denote a function of a region $\mathrm{G}$ in time t. So, let us understand $R(G, t)$ as the set $\{R(p, t)\}, \forall p \in G$.

We will then call the tuple 


$$
\sigma(p, t)=\left(G^{s}(p), R\left(G^{s}(p), t\right)\right)
$$

as being a Signal located in place p.

Given these premises, we may now proceed to define a Mathematical Semiosis. Mathematical Semiosis is a process where a signal $\sigma_{s}\left(p_{s}, t_{s}\right)$ is said to represent another signal $\sigma_{o}\left(p_{o}, t_{o}\right)$. The process consolidates when $\sigma_{s}\left(p_{s}, t_{s}\right)$ is used to generate another signal, $\sigma_{i}\left(p_{i}, t_{i}\right)$, for $t_{i}>t_{s}$. In this case, $\sigma_{s}\left(p_{s}, t_{s}\right)$ is said to be a Sign of $\sigma_{o}\left(p_{o}, t_{o}\right)$, which is called to be its Object, and $\sigma_{i}\left(p_{i}, t_{i}\right)$ is said to be the Interpretant of the sign. But this is not enough that $\sigma_{i}\left(p_{i}, t_{i}\right)$ to be generated by $\sigma_{s}\left(p_{s}, t_{s}\right)$. In order for this to be a semiosis, there is a further condition. And this condition is that $\sigma_{i}\left(p_{i}, t_{i}\right)$ should maintain the relation that $\sigma_{s}\left(p_{s}, t_{s}\right)$ had to $\sigma_{o}\left(p_{o}, t_{o}\right)$. In other words, it should be able to generate a further interpretant, that should also maintain this relation to the object. This is the way we guarantee that $\sigma_{s}\left(p_{s}, t_{s}\right)$ really "represents" $\sigma_{o}\left(p_{o}, t_{o}\right)$.

This process is basically performed by the agents in the mathematical universe, and do have its realization within its mathematical reality, in possible different places and times.

So, Mathematical Semiosis is a process by which an agent reads a signal from a Mathematical Reality, and generates output to this same Mathematical Reality in a future time. The effect produced by the Agent is the Interpretant of the sign.

The most simple kind of semiosis is the copy. In this case, the signal $\sigma_{s}\left(p_{s}, t_{s}\right)$ is an exact copy of $\sigma_{o}\left(p_{o}, t_{o}\right)$, for a possible different place and time. In being a copy, we may assure that it is always possible to generate another copy $\sigma_{i}\left(p_{i}, t_{i}\right)$ in a different place and time in the future. So, an exact copy is the most simple representation of something. But there may be more sophisticated kinds of representation. This copy should not be an exact copy, but just share a partial set of attributes. Both the copy and a partial copy will be called "icons". But there may be the case that $\sigma_{s}\left(p_{s}, t_{s}\right)$ and $\sigma_{o}\left(p_{o}, t_{o}\right)$ do not share any kind of attribute in common. But even in this case, they may give rise to a process of semiosis. By using the mathematical reality in which $\sigma_{s}\left(p_{s}, t_{s}\right)$ is realized in order to obtain either $\sigma_{o}\left(p_{o}, t_{o}\right)$ or a copy of it. In this case, proximity in space (place) and time could be used to create the interpretant. This kind of signs are called indexes. But there may be a more radical kind of sign, where there may be no sharing of attributes nor a space-time connection between sign and object. This kind of sign is called a symbol by Peirce. To understand how symbols are possible is a very challenging exercise in the interpretation of Peirce's work. A symbol, according to Peirce, is a lawfull or conventional connection between a sign and an object. But, if it is conventional, how can an agent generate an interpretant, that is still related to this same object ? We don't have an answer to this challenge, in a strict Peircean view. But we have some hypothesis on how to solve this puzzle. A possible way of solving this, is to allow the agents in the mathematical universe to have inner mathematical realities. An inner mathematical reality is just like a mathematical reality in the mathematical universe, but instead of being a shared space and time, (i.e. Places where all the agents are able to perceive and actuate) are internal, private instances where only the own agents are able to perceive and act. So, if we consider this inner mathematical reality, in addition to the standard mathematical reality, we may have a clue on how symbols are possible. When Peirce says that a symbol is a convention, i.e., that there may be no sharing of attributes and no physical connection (in space and time) between the sign and the object, he may be considering this statement related only to the standard reality, the one which in our case is shared by all the agents - the mathematical reality of our mathematical universe. So, considering just this mathematical reality, there is no sharing of attributes neither a physical connection in terms of space and time. But, there may be either a sharing of attributes or a physical connection in terms of space and time, considering this inner mathematical reality that each agent should possess. This may solve the puzzle and may allow for the construction of symbol-processing capabilities by our agents.

\section{CONCLUSION}

This paper was written with the aim of putting forward a first sketch of a mathematical framework which should allow us to discuss the possibility of artificial agents to be fully capable of making meaning of their surrounding world. In order to obtain this, we recommend the use of Peircean semiotics as the main theory to ground the concepts of representation and meaning, and provided a mathematical abstraction of this theory with the aim of making viable the creation of such meaning machines.

Despite the existence of other mathematical models for the semiotic phenomena, we felt they were not adequate in order to build artificial agents fully capable of being meaning machines. Burch's model is more concerned with some particular aspects of Peirce's theory, and not in modelling the process of semiosis. Goguen's model is not strictly Peircean, and more suitable to be used in applications of user interface design than in artificial intelligence. Marty's model, despite being very precise in its correlation to Peirce's theory is too much complex to be easily used in order to generate an architecture for an artificial agent capable of performing meaning processes.

The model proposed here is at the same time quite simple, strict to the most basic Peircean principles, and up to our judgment, very suitable to be used for the construction of prototypes of simple meaning machines.

\section{REFERENCES}

[1] Gudwin, R.R., Queiroz, J. (eds.) - Semiotics and Intelligent Systems Development, Idea Group Publishers, 2006. 
[2] Antônio Gomes, Ricardo Gudwin, João Queiroz - "Towards Meaning Processes in Computers from Peircean Semiotics" - S.E.E.D. Journal (Semiotics, Evolution, Energy, and Development), Volume 3, No. 2, November 2003, SEED 2003 (2), p. 69-79

[3] Antônio Gomes, Ricardo Gudwin, João Queiroz - "Meaningful Agents: A Semiotic Approach" - Proceedings of the 2005 IEEE KIMAS'05, 1821 April 2005, Waltham, MA, USA, pp. 399-404.

[4] R. R. Gudwin - Semiotic Synthesis and Semionic Networks S.E.E.D. Journal (Semiotics, Evolution, Energy, and Development), Volume 2, No. 2, August 2002, p. 55-83.

[5] João Queiroz and Ricardo Gudwin - Computational Intelligence and Semiotics - On the Synthesis of Semiosis in Artificial Systems and Creatures - Editorial - Special Issue of S.E.E.D. Journal (Semiotics, Evolution, Energy, and Development), Volume 3, No. 3, December 2003.

[6] Angelo Loula, Ricardo Gudwin, João Queiroz - Synthetic Approach of Symbolic Creatures - S.E.E.D. Journal (Semiotics, Evolution, Energy, and Development), Volume 3, No. 3, December 2003, SEED 2003 (3), p. $125-133$

[7] Queiroz, J., Gudwin, R., Loula, A. Semiótica sintética -- sobre a síntese de criaturas simbólicas artificiais. In Cognito 3(2) 2006.

[8] Ribeiro, S., Loula, A., Araújo, I., Gudwin, R. \& Queiroz, J.- Symbols are not uniquely. Biosystems, (in press).

[9] Marty, Robert - "Foundations of a mathematícal semiotics", Mathematical Aspects Of Natural And Formal Languages, G. Paun ed., World Scientific Series in Computer Science, Vol.43 (1994), World Scientific Publishing Co. Pte.Ltd. Singapore, 257-273.

[10] Marty, Robert - "Les fondements algébriques de la sémiotique", DEGRES, n54-55, (1988), A. Helbo éd., Bruxelles, b-b16.

[11] Marty, Robert - "Champs d'interprétants : un modèle formalisé de la sémiosis", Semiotic Theory And Practice (1988), Mouton de Gruyter, Berlin-New-York-Amsterdam, pp. 729-737.

[12] Marty, Robert - L'algebre Des Signes, (1990), Collection "Foundations of Semiotics", John Benjamins, Amsterdam/Philadelphie, 406 p.

[13] Joseph Goguen - "Semiotic Morphisms, Representations, and Blending for User Interface Design” - Keynote lecture, in Proceedings, AMAST Workshop on Algebraic Methods in Language Processing, edited by Fausto Spoto, Giuseppi Scollo and Anton Nijholt, AMAST Press, 2003, pages 1-15. Workshop held Verona, Italy, 25 - 27 August 2003

[14] Joseph Goguen and D. Fox Harrell - "Information Visualization and Semiotic Morphisms" - in Multidisciplinary Approaches to Visual Representations and Interpretations, ed. Grant Malcolm (Elsevier 2004), pages 93-106.

[15] Joseph Goguen - "An Introduction to Algebraic Semiotics, with Applications to User Interface Design", in Computation for Metaphor, Analogy and Agents, edited by Chrystopher Nehaniv, Springer Lecture Notes in Artificial Intelligence, volume 1562, 1999, pages 242-291

[16] Robert W. Burch - A Peircean Reduction Thesis: the Foundations of Topological Logic. Lubbock, Texas: Texas Tech University Press, 1991.

[17] Robert W. Burch - "Valental Aspects of Peircean Algebraic Logic" - In: Computers Math. Applic. Vol.23, No.6-9, pp.665-677, 1992.

[18] Stephan Wolfram. Cellular Automata and Complexity: Collected Papers. Addison-Wesley, 1994. ISBN 0-201-62664-0

[19] Robert Rosen - Anticipatory Systems - Pergamon Press, 1985.

[20] Mihai Nadin - "Anticipation-A Spooky Computation” - Third International Conference on Computing Anticipatory Systems (CASYS 99), HEC, Liege, Belgium, August 9-14, 1999.

[21] Mihai Nadin - Anticipation-The End Is Where We Start From (EnglishGerman-French text). Baden, Switzerland: Lars Müller Publishers, 2003, 150 pp. 


\section{Copyright Information}

(C) 2007 IEEE. Personal use of this material is permitted. However, permission to reprint/republish this material for advertising or promotional purposes or for creating new collective works for resale or redistribution to servers or lists, or to reuse any copyrighted component of this work in other works must be obtained from the IEEE. 